ARTICLE OPEN

Check for updates

\title{
Robust energy-selective tunneling readout of singlet-triplet qubits under large magnetic field gradient
}

\author{
Wonjin Jang ${ }^{1,4}$, Jehyun Kim ${ }^{1,4}$, Min-Kyun Cho ${ }^{1}$, Hwanchul Chung ${ }^{2}$, Sanghyeok Park', Jaeun Eom¹, Vladimir Umansky ${ }^{3}$, \\ Yunchul Chung ${ }^{2}$ and Dohun Kim (iD)
}

Fast and high-fidelity quantum state detection is essential for building robust spin-based quantum information processing platforms in semiconductors. The Pauli spin blockade (PSB)-based spin-to-charge conversion and its variants are widely used for the spin state discrimination of two-electron singlet-triplet $\left(\mathrm{ST}_{0}\right)$ qubits; however, the single-shot measurement fidelity is limited by either the low signal contrast, or the short lifetime of the triplet state at the PSB energy detuning, especially due to strong mixing with singlet states at large magnetic field gradients. Ultimately, the limited single-shot measurement fidelity leads to low visibility of quantum operations. Here, we demonstrate an alternative method to achieve spin-to-charge conversion of $\mathrm{ST}_{0}$ qubit states using energy-selective tunneling between doubly occupied quantum dots (QDs) and electron reservoirs. We demonstrate a single-shot measurement fidelity of $90 \%$ and an S-To oscillation visibility of $81 \%$ at a field gradient of $100 \mathrm{mT}\left(\sim 500 \mathrm{MHz} h\left(g^{*} \cdot \mu_{B}\right)^{-1}\right)$; this allows single-shot readout with full electron charge signal contrast and, at the same time, long and tunable measurement time with negligible effect of relaxation even at strong magnetic field gradients. Using an rf-sensor positioned opposite to the QD array, we apply this method to two $\mathrm{ST}_{0}$ qubits and show high-visibility readout of two individual single-qubit gate operations is possible with a single if single-electron transistor sensor. We expect our measurement scheme for two-electron spin states can be applied to various hosting materials and provides a simplified and complementary route for multiple qubit state detection with high accuracy in QD-based quantum computing platforms.

npj Quantum Information (2020)6:64; https://doi.org/10.1038/s41534-020-00295-w

\section{INTRODUCTION}

The assessment of general quantum information processing performance can be divided into that of state initialization, manipulation, and measurement. Rapid progress has been made in semiconductor quantum dot (QD) platforms, with independent demonstrations of, for example, high-fidelity state initialization of single and double QD spin qubits ${ }^{1-3}$, high-fidelity quantum control with resonant microwaves $^{4-8}$ and non-adiabatic pulses $^{1,9,10}$, and high-fidelity state measurements using spin-tocharge conversion ${ }^{3,11-19}$. However, the high visibility of a quantum operation requires high fidelity in all stages of the quantum algorithm execution, which has been demonstrated in only a few types of spin qubits so $\mathrm{far}^{4,6,7,10,20,21}$.

For double QD two-electron spin qubits, the Pauli spin blockade (PSB) phenomenon is typically used for discriminating spin-singlet (S) and -triplet $\left(T_{0}\right)$ states where different spin states are mapped according to the difference in the relative charge occupation of two electrons inside the double QD, which is detected by a nearby electrometer ${ }^{22-24}$. As the spin-dependent signal deterministically appear at the measurement phase defined by the pulse sequence at the PSB, the measurement window can be shortened to the limit which allows enough signal to noise ratio (SNR) to discriminate the different spin signal ${ }^{13}$, and such can lead to high measurement bandwidth. However, depending on the device design, the signal contrast can be small compared to the signal of one electron, especially when the charge sensor position in the device is not aligned with the QD axis. This issue is particularly problematic in recent multiple QD designs ${ }^{25-29}$, where the charge sensor positioned opposite to the qubit array increases the range of QDs detectable by one sensor, but renders sensitive measurement of the relative electron position between nearest-neighbor QDs difficult.

Moreover, the spatial magnetic field difference $\Delta B_{/ /}=\mid \Delta B_{\mathrm{L} / /}-$ $B_{\mathrm{R} / /}$, where the $B_{\mathrm{L} / /}\left(B_{\mathrm{R} / /}\right)$ denotes the magnetic field strength parallel to the spin quantization axis at the left (right) dot, provides relaxation pathways through $(1,1) \mathrm{T}_{0}-(1,1) \mathrm{S}$ mixing and rapid $(1,1)$ $\mathrm{S}-(2,0) \mathrm{S}$ tunneling in the PSB region as shown in the solid green regions in Fig. 1a, and normal PSB readout is difficult under large $\Delta B_{/ /}$. For example when $\Delta B_{/ /}>200 \mathrm{MHz} h\left(g^{*} \cdot \mu_{B}\right)^{-1}$, where $h$ is the Planck's constant, $g^{*}$ is the electron $g$-factor in GaAs, and $\mu_{B}$ is the Bohr magneton, the fast spin relaxation is known to lead to vanishing oscillation visibility ${ }^{30}$. As most QD spin qubit platforms utilize sizeable intrinsic ${ }^{2,31,32}$ or extrinsic ${ }^{33} \Delta B_{/ /}$to realize individual qubit addressing and high-fidelity single- and two-qubit operations $4,6,34,35$, it is important to develop fast readout techniques that enable high-fidelity spin detection even at large $\Delta B_{/ /}$. So far, visibility higher than $95 \%$ using PSB readout can be achieved only for small $\Delta B_{/ /}$despite the method's high measurement bandwidth ${ }^{13,18}$.

These limitations of conventional PSB readout have been addressed in previous works, and several variants of the PSB readout have been developed for various QD systems ${ }^{14-17}$. In the latched readout scheme ${ }^{14}$, the lack of the reservoir on one side of the double QD enables spin conversion to the $(1,0)$ or $(2,1)$ charge state, enhancing the signal contrast. In ref. ${ }^{15}$, singlet-triplet $\left(\mathrm{ST}_{0}\right)$ qubit readout was performed in a triple QD to isolate the middle QD from the reservoirs, and the qubit state conversion to a metastable charge state enabled robust, high-fidelity qubit

\footnotetext{
Department of Physics and Astronomy, and Institute of Applied Physics, Seoul National University, Seoul 08826, Korea. ${ }^{2}$ Department of Physics, Pusan National University, Busan 46241, Korea. ${ }^{3}$ Braun Center for Submicron Research, Department of Condensed Matter Physics, Weizmann Institute of Science, Rehovot 76100 , Israel. ${ }^{4}$ These authors contributed equally: Wonjin Jang, Jehyun Kim. ${ }^{凶}$ email: dohunkim@snu.ac.kr
} 
a

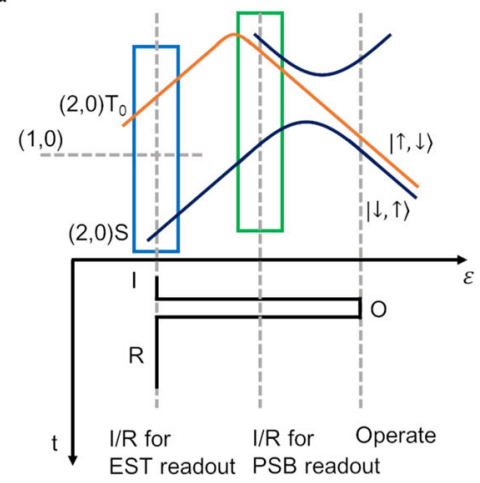

EST readout PSB readout
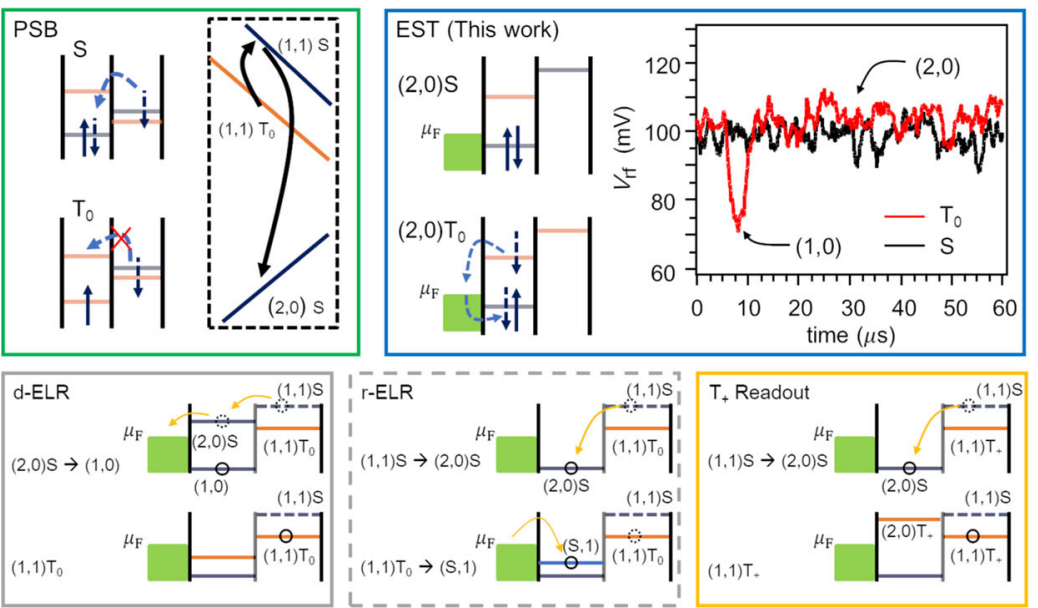

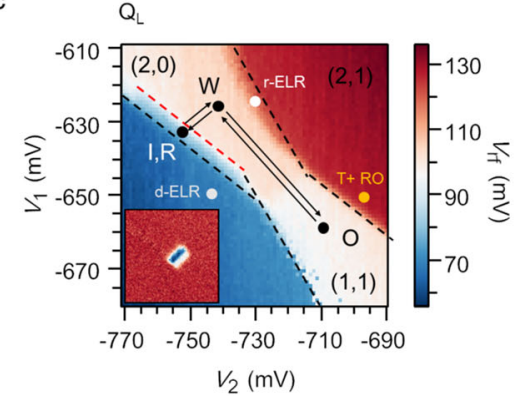

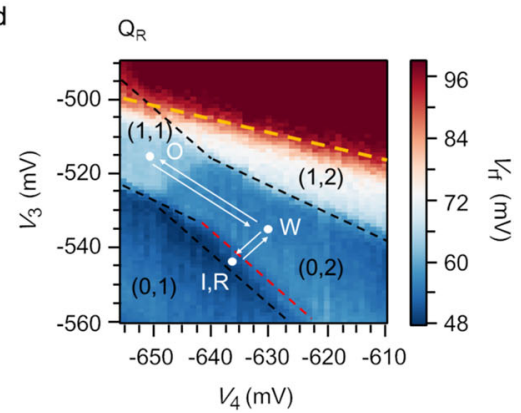

Fig. 1 Energy levels and device platform. a. Schematic of the singlet-triplet (ST $\mathrm{ST}_{0}$ ) qubit energy levels as a function of detuning $\varepsilon$ with energyselective tunneling (EST, blue boxes)- and Pauli spin blockade (PSB, green boxes)-based readout schemes. Green panel: At the PSB readout point, the $(1,1) \mathrm{S}$ state tunnels into the $(2,0)$ charge state while the tunneling from the $(1,1) \mathrm{T}_{0}$ state is blocked. The relative charge position is observed to determine the qubit state. Finite magnetic field difference $\Delta B / /$ provides a relaxation pathway for the $(1,1) \mathrm{T}_{0}$ state. Blue panel: The Fermi level resides between the $(2,0) \mathrm{S}$ and $(2,0) \mathrm{T}_{0}$ states, which enables EST. The triplet state (red) tunnels out to the $(1,0)$ and initializes to the $(2,0) \mathrm{S}$, while no tunneling occurs for the $\mathrm{S}$ state. For comparison, three other types of modified PSB readout scheme are presented. Gray panel: In the direct enhanced latched readout (d-ELR) scheme, the $(2,0) S$ state tunnels out to $(1,0)$ while the spin-blockaded $(1,1) \mathrm{T}_{0}$ state cannot tunnel out ${ }^{14}$. Dashed gray panel: At the reverse ELR ( $r$-ELR) point, an electron tunnels into the spin-blockaded $T_{0}$ state to form the $(S, 1)$ while the $S$ state stays at the $(2,0)^{14,15,17}$. Yellow panel: In the $T_{+}$readout scheme ${ }^{16}$, one of the qubit states is conversed into the $T_{+}$state to prevent the relaxation in the PSB. The readout is taken in the PSB by discriminating the $(2,0) \mathrm{S}$ and $(1,1) \mathrm{T}_{+}$. Points corresponding to different schemes are denoted in Fig. 1c. b Scanning electron microscopy image of the device. Green (orange) dots indicate the left (right) $\mathrm{ST}_{0}$ qubit $\mathrm{Q}_{\mathrm{L}}\left(\mathrm{Q}_{\mathrm{R}}\right)$, and the yellow dot indicates the rf single-electron transistor (rf-set). The blue arrow indicates the external magnetic field direction. The white scale bar corresponds to $500 \mathrm{~nm}$. c, d Charge stability diagram for $Q_{L}\left(Q_{R}\right)$ operation with the pulse cycling $I-W-O-W-R$ points superimposed. The red dashed line shows the boundary of the region inside which the EST readout is appropriate. The inset of $\mathbf{c}$ shows the PSB readout signal for the same area observed by gated integration. The yellow line in $\mathbf{d}$ shows the electron transition signal of the QD coupled to $V_{2}$.

readout. While these techniques enhance the signal contrast to the full electron charge, the explicit demonstration of such methods combined with high-fidelity operation under large $\Delta B_{/ /}$ $\left(>200 \mathrm{MHz} h\left(g^{*} \cdot \mu_{B}\right)^{-1}\right.$ ) has not been reported to date. We stress that it is unclear whether the readout near the $(2,1)$ charge transition ${ }^{15,17}$ will not suffer from the fast $T_{0}$ relaxation if the spin mixing rate due to $\Delta B_{/ /}$is comparable to the $(1,1) T_{0}-(2,1)$ tunneling rate. We note here that unlike the readout methods near the $(2,1)$ charge transition ${ }^{15,17}, \mathrm{~T}_{0}$ relaxation pathway is inherently absent at the readout position of this work, as both the $\mathrm{S}$ and $\mathrm{T}_{0}$ state occupy the $(2,0)$ charge state as we describe below in detail. On the other hand, Orona, L. A. et al. ${ }^{16}$ reported the shelving readout technique, whereby one of the qubit states is first converted to the $T_{+}$state through fast electron exchange with the reservoir to prevent mixing with the $(1,1) S$ state, enabling high-visibility readout of the $\mathrm{ST}_{0}$ spin qubit. They showed explicitly that single-shot readout is possible even for $\Delta B_{/ /} \sim 180 \mathrm{mT}(\sim 900$ $\left.\mathrm{MHz} h\left(g^{*} \cdot \mu_{B}\right)^{-1}\right)$ by optimizing the shelving pulse sequence. However, the technique relies on PSB for final spin-to-charge conversion and is expected to be effective only when the charge sensor is sensitive to the relative position of electrons in the double QD.
Here, we demonstrate the energy-selective tunneling (EST) readout, commonly called Elzerman readout ${ }^{11}$, of $\mathrm{ST}_{0}$ qubits under large $\Delta B_{/ /}$, accomplishing both signal enhancement, due to oneelectron tunneling, and long measurement time, enabling a robust single-shot readout. Unlike previous works, which demonstrated independent enhancement of the signal contrast and measurement time through intermediate spin or charge state conversion steps, our scheme does not require additional state conversion during the readout. Using large voltage modulation by rapid pulsing with $\varepsilon$ ranging from the PSB-lifted $(2,0)$ to the deep $(1,1)$ charge regions, where the exchange coupling $J(\varepsilon)$ is turned off, we explicitly demonstrate a single-shot measurement fidelity of $90 \pm$ $1.3 \%$ and an $\mathrm{S}-\mathrm{T}_{0}$ oscillation visibility of $81 \%$ at $\Delta B_{/ /} \sim 100 \mathrm{mT}$, corresponding to an oscillation frequency of $500 \mathrm{MHz}$. Furthermore, we demonstrate the detection of coherent operation of two individual $\mathrm{ST}_{0}$ qubits in a quadruple $\mathrm{QD}$ array with a single rfreflectometry line. We stress that we combine previous methods, which individually demonstrated the Elzerman readout of the twoelectron spin states ${ }^{12}$, large $\Delta B_{/ /}$generation with micromagnet ${ }^{33}$, high-fidelity control of the $\mathrm{ST}_{0}$ qubit $^{36}$, and robust measurement within a single quantum processor yielding a record high quantum oscillation visibility in large $\Delta B_{/ /}$. We also note that this 
is achieved at the expense of high bandwidth of PSB readout due to EST readout's intrinsic timing uncertainty in tunneling events. However the achieved measurement time on the order of $100 \mu \mathrm{s}$ in this work using EST readout is still useful for future application to fast spin state readout, for example single-shot readout-based Bayesian estimation ${ }^{37}$. In this paper, we describe the proposed EST readout method in detail, compare it with the conventional PSB readout, and suggest possible routes for its further optimization.

\section{RESULTS}

Energy-selective tunneling readout

The blue rectangular regions in Fig. 1a show the position of $\varepsilon$ and the energy level configuration used for EST state initialization and readout. At this readout point, the PSB is lifted, and both $S$ and $T_{0}$ levels can first occupy the $(2,0)$ charge state, the energies of which are separated by $\mathrm{ST}_{0}$ splitting typically in the order of $\sim 25-30 \mathrm{GHz}^{38}$, depending on the dot-confining potential. Near the $(1,0)-(2,0)$ electron transition, the electrochemical potential of the reservoir resides between these states, which enables the EST of the $\mathrm{ST}_{0}$ qubits. As discussed in detail below, we observe the single-shot spin-dependent tunneling signal where one electron occupying an excited orbital state of the $(2,0) T_{0}$ state tunnels to the reservoir to form the $(1,0)$ charge state, leading to an abrupt change in the sensor signal, and predominantly initializes back to the energetically favorable $(2,0) S$ state. In contrast, no tunneling occurs for the $(2,0) \mathrm{S}$ state (see Fig. 1a, blue right panel).

We study a quadruple QD array with an rf single-electron transistor (rf-set) sensor consisting of $\mathrm{Au} / \mathrm{Ti}$ metal gates on top of a $\mathrm{GaAs} / \mathrm{AlGaAs}$ heterostructure, where a $2 \mathrm{D}$ electron gas (2DEG) is formed $\sim 70 \mathrm{~nm}$ below the surface (Fig. 1b). A 250-nm thick rectangular Co micromagnet with large shape anisotropy was deposited on top of the heterostructure to generate stable $\Delta B_{/ /}$for $\mathrm{ST}_{0}$ qubit operation ${ }^{33,36,39,40}$ (see methods section for fabrication details). The device was placed on a plate in a dilution refrigerator at $\sim 20 \mathrm{mK}$ and an in-plane magnetic field $B_{z, \text { ext }}$ of $225 \mathrm{mT}$ was applied. To demonstrate the EST readout in the experiment, we independently operated and readout two $S T_{0}$ qubits $\left(Q_{L}\right.$ and $\left.Q_{R}\right)$ in the noninteracting regime by blocking $Q_{L}-Q_{R}$ tunneling using appropriate gate voltages. We monitored the rf-reflectance of the rf-set sensor (Fig. 1b, yellow dot) for fast single-shot charge occupancy detection in the $\mu$ s time scale $e^{41,42}$. The intra qubit tunnel couplings for both $\mathrm{Q}_{L}$ and $\mathrm{Q}_{R}$ were tuned above $8 \mathrm{GHz}$ to suppress unwanted Landau-Zener-Stuckelberg interference under fast $\varepsilon$ modulation, and we estimated the electron temperature to be $\sim 230 \mathrm{mK}$ (see also Supplementary Note 1).

We first locate appropriate EST readout points in the charge stability diagrams. Figure 1c (1d) shows the relevant region in the stability diagram for the $\mathrm{Q}_{\mathrm{L}}\left(\mathrm{Q}_{\mathrm{R}}\right)$ qubit operation as a function of two gate voltages $V_{1}\left(V_{3}\right)$ and $V_{2}\left(V_{4}\right)$. We superimpose the cyclic voltage pulse, sequentially reaching I-W-O-W-R points in the stability diagram (see Fig. 1C, d) with a pulse rise time of 200 ps. During the transition from the point $\mathrm{W}$ to point $\mathrm{O}$ stage, the pulse brings the initialized $(2,0) S$ state to the deep $(1,1)$ region nonadiabatically, and the time evolution at point $O$ results in coherent $\mathrm{S}-\mathrm{T}_{0}$ mixing due to $\Delta B_{/ /}$. The resultant non-zero $\mathrm{T}_{0}$ probability is detected at the $I / R$ point. For this initial measurement, the duration of each pulse stage was not strictly calibrated, but the repetition rate was set to $10 \mathrm{kHz}$. The resulting 'mouse-bite' pattern inside the $(2,0)$ charge region (Fig. 1c., boundary marked by the red dashed line) implies the $(1,0)$ charge occupancy within the measurement window, which arises from the EST of the $\mathrm{ST}_{0}$ qubit states averaged over $100 \mu \mathrm{s}$. For comparison, we note that the PSB readout signal with a similar pulse sequence is not clearly visible in the main panel of Fig. $1 \mathrm{c}$ in the time-averaged manner due to fast relaxation, as described above. The inset in Fig. 1c shows the PSB readout signal measured by gated (boxcar) integration (see Supplementary Note 2), where an approximately 100 ns gate window was applied immediately after the pulse sequence. This difference in the available range of measurement time scale clearly contrasts two distinct readout mechanisms for the spin-to-charge conversion of $\mathrm{ST}_{0}$ qubits.

The PSB and EST readouts are systematically compared through time-resolved relaxation measurements, which also serve as calibration of the readout parameters for EST readout visibility optimization. Figure $2 \mathrm{a}$, b shows the relaxation of the sensor signal as a function of waiting time $\tau$ before reaching the measurement stage, using the pulse sequence shown in the inset of Fig. $2 a, b$ near the PSB (EST) readout position for $Q_{L}$ (see Supplementary Note 3 for measurement result and fidelity analysis of $Q_{R}$ ). As expected, the lifetime $T_{1}$ of the $T_{0}$ state at the PSB region is in the order of $200 \mathrm{~ns}$, indicating strong spin state mixing and subsequent charge tunneling due to the large $\Delta B_{/ /}$produced by the micromagnet (see Supplementary Note 4 for magnetic field simulation). However, at large negative $\varepsilon$, the PSB is eventually lifted, and the absence of rapid spin mixing as well as the insensitivity of the $(2,0) \mathrm{T}_{0}-(2,0) \mathrm{S}$ spin splitting to charge fluctuations ensures the long lifetime of the $T_{0}$ state. The evolution time at $\mathrm{O}$ is varied in the EST relaxation time measurement in Fig. $2 \mathrm{~b}$, and the amplitude decay of the coherent oscillation is probed to remove background signals typically present for long pulse repetition periods. The resultant $T_{1}$ of $337 \mu$ s is three orders of magnitude longer than that in PSB readout. Without fast $\varepsilon$ modulation, a long $T_{1}$ exceeding $2.5 \mathrm{~ms}$ has been reported in $\mathrm{GaAs}$ $\mathrm{QDs}^{43}$ implying that further optimization is possible.

\section{Measurement fidelity optimization}

Next, we discuss the calibration of the tunnel rates for single-shot readout and the optimization of the readout fidelity and visibility with the given experimental parameters. While for time-averaged charge detection we use a minimum integration time of $30 \mathrm{~ns}$ in the signal demodulation setup, corresponding to a measurement bandwidth of $33 \mathrm{MHz}$, we set the integration time to $1 \mu \mathrm{s}$ for single-shot detection to increase the signal to noise ratio, and we typically tune the tunneling rates to less than $1 \mathrm{MHz}$. Figure $2 \mathrm{c}$ shows time-resolved tunnel out events triggered by the end of the pulse sequence from which we measure the tunneling out rate $\nu_{\text {out }} \sim \tau_{\text {out }}^{-1}=(16 \mu \mathrm{s})^{-1}$, extracted from the fit to an exponentially decaying function. The rate is within our measurement bandwidth. Also note that the ratio $T_{1} / \tau_{\text {out }}$ is at least 20 , which is reasonable to perform high-fidelity measurements above $90 \%{ }^{44}$. Figure $2 \mathrm{~d}$ shows the resultant histogram showing a separation of the mean value of the $S$ and $T_{0}$ signal levels of more than 8 times the standard deviation, confirming the high fidelity of single-shot spin state detection with $1 \mu \mathrm{s}$ integration time. We also find good agreement between the experimental and numerically simulated single-shot histograms ${ }^{3}$ generated using the measured tunneling rates and signal to noise ratio (See Supplementary Note 5 for details).

After the rf demodulation stage, we further apply correlated double sampling $(C D S)^{15}$ to the single-shot traces to simplify the state discrimination and measurement automation. Using a fast boxcar integration with two gate windows that are $5 \mu \mathrm{s}$ apart in the time domain, a dc background-removed pseudo-time derivative of the single-shot traces is generated, enabling separate detection of tunneling out/in events with an external pulse counter (Stanford Research Systems, SR400 dual gated photon counter) and time-correlated pulse counting with a multichannel scaler (Stanford Research Systems, SR430 multichannel scaler) without the need for customized field-programmable gate array (FPGA) programming ${ }^{37,45}$ (see Supplementary Note 2 for details of the CDS scheme). While this scheme was successful, the electronic measurement bandwidth was further reduced to $200 \mathrm{kHz}$ for single-shot detection, which resulted in a relatively long readout 
a

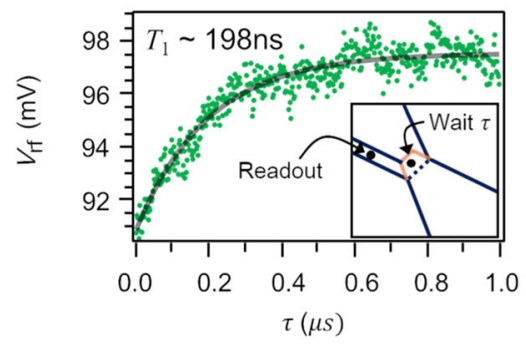

b

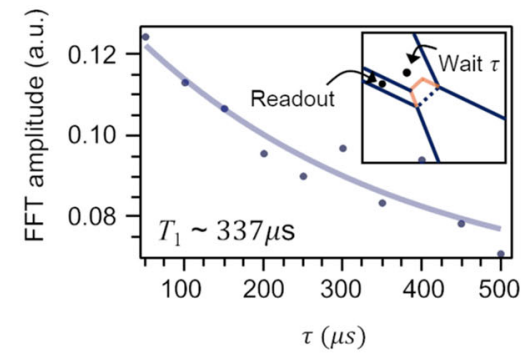

C

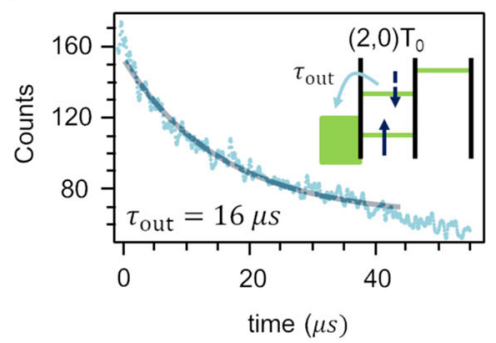

d

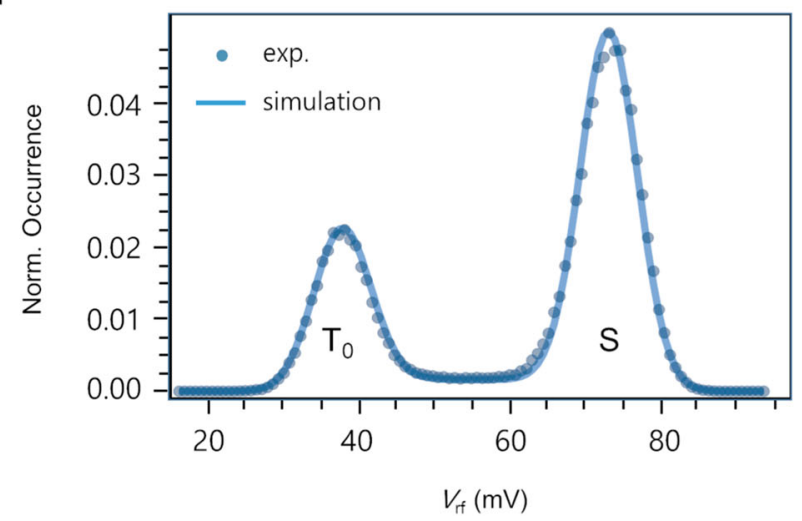

e

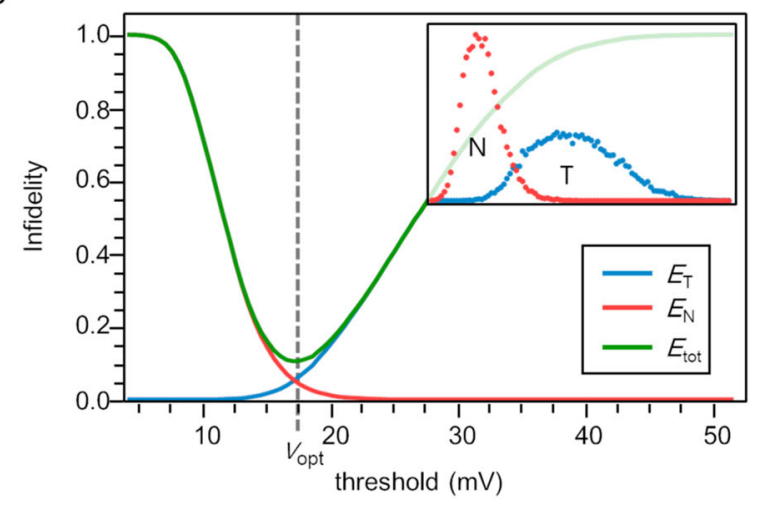

Fig. 2 Time-resolved relaxation measurements and fidelity analysis of $\mathbf{Q}_{\mathbf{L}}$. a. Relaxation time measurement at PSB readout. The timeaveraged rf-demodulated signal $V_{\mathrm{rf}}$ is recorded $\times$ as a function of the waiting time $\tau$ at the $\varepsilon$ denoted in the inset. $T_{1} \sim 200 \mathrm{~ns}$ is extracted from the fitting data to the exponential decay curve. b Relaxation time measurement near EST readout. The decay of the coherent oscillation is observed along the waiting time $\tau$ near the detuning at the measurement point denoted in the inset. $T_{1} \sim 337 \mu$ s is extracted. c Histogram of the tunneling out events triggered by the end of the manipulation pulse as a function of time. $\mathbf{d}$ Histogram of the experimental and simulated rf-demodulated single-shot traces with the application of $\pi$ pulses for EST readout showing a mean value separation of more than 8 times the standard deviation. e Tunneling detection infidelity calculated from the CDS peak amplitude histogram shown in the inset. Minimum total error $\left(E_{\mathrm{T}}+E_{\mathrm{N}}\right)$ of $\sim 10.5 \%$ corresponding to $E_{\mathrm{T}} \sim 5 \%$, and $E_{\mathrm{N}} \sim 5.5 \%$ are estimated at the optimal threshold voltage $V_{\mathrm{opt}}$.

time requiring relatively slow tunneling rates. To simulate realistic measurement conditions, we applied the numerical CDS filter to the simulated single-shot traces (Fig. 2e) and reproduced the tunneling detection fidelity of the measurement setup. As the measured electron temperature $\left(230 \mathrm{mK} \sim 5-6 \mathrm{GHz} / \mathrm{k}_{B}\right.$, where $\mathrm{h}$ is the Planck's constant and $k_{B}$ is the Boltzmann's constant) compared to the $\mathrm{ST}_{0}$ splitting $(25-30 \mathrm{GHz})$ may trigger unwanted events such as false initialization, thermal tunneling of the ground state, and double-tunneling events within the measurement windows, we have introduced corresponding thermal parameters to the analysis. The parameters were utilized to model the Larmor oscillation measured (see Fig. 3 ), and the values were extracted from the least squares fitting with the experimental data to yield the final measurement fidelity (see Supplementary Note 5 for measurement fidelity analysis). The resulting theoretical measurement fidelity of the $\mathrm{Q}_{\mathrm{L}}$ is $90 \pm 1.3 \%$, corresponding to a visibility of $80 \pm 2.6 \%$, confirming that high-fidelity single-shot detection is possible at the given experimental conditions. Moreover, in Supplementary Note 6, we show through numerical simulation that FPGA-based single-shot detection, which we plan to perform in the future, will yield a measurement fidelity (visibility) of $94 \%$ $(89 \%)$ at the same experimental condition through faster and more accurate peak detection, which lowers the tunneling detection infidelity.

High-visibility quantum control with the EST readout

We now demonstrate high-visibility coherent qubit operations with the EST single-shot readout. The panels in Fig. 3 show the high-visibility two-axis control of $Q_{L}$ (Fig. 3a-c) and $Q_{R}$ (Fig. 3d-f) under large $\Delta B_{/ /}$recorded with a single rf-set. For the $\Delta B_{/ /}$ oscillations (Fig. 3a, d), the I-W-O-W-R with the period of $150 \mu \mathrm{s}$
(Fig. 3a, top panel) was applied, and the evolution time at $O$ was varied from 0 to $10 \mathrm{~ns}$. Each trace in Fig. $3 \mathrm{a}$, $\mathrm{d}$ is the average of 50 repeated measurements with 2000 shots per point, which takes over $5 \mathrm{~min}$; thus, we expect an ensemble-averaged coherence time of $\mathrm{ST}_{0}$ qubit oscillation $T_{2}^{*}$ in the order of $15 \mathrm{~ns}$, limited by nuclear bath fluctuation ${ }^{1}$. We clearly observe coherent oscillations of $\mathrm{Q}_{\mathrm{L}}\left(\mathrm{Q}_{\mathrm{R}}\right)$ with $\sim 81 \%(\sim 64 \%)$ visibility, which is consistent with the results of the numerical simulation reported in Supplementary Note 5 . Under the large $\Delta B / /$ of 100 (80) $\mathrm{mT}$, corresponding to an oscillation frequency of $500(400) \mathrm{MHz}$, we expect the Q-factor $\left(T_{2}^{*} / T_{\pi}\right)$ of the oscillation to reach up to 28 (22) for $\mathrm{Q}_{\mathrm{L}}\left(\mathrm{Q}_{\mathrm{R}}\right)$, even with the measured ensemble-averaged $T_{2}^{*} \sim 15 \mathrm{~ns}$. Moreover, we estimate the leakage probability during the fast ramp less than $2 \%$ (see Supplementary Note 7); thus, we assume here that the effect of leakage error to the visibility is not significant. As discussed above, electronic bandwidth owing to the CDS technique is one of the factors limiting the visibility for both $Q_{L}$ and $Q_{R}$. Moreover, we estimate about $8 \%(9 \%)$ probability that the ground state tunnel out to the reservoir and $4 \%(2 \%)$ probability of false initialization to $T_{0}$ state for $Q_{L}\left(Q_{R}\right)$, showing that the reduction of the measurement fidelity and visibility in our experiment stems from the combination of the thermal effects, spin relaxation, and electronic bandwidth of the CDS method. For $Q_{R}$, tuning to an even longer $\tau_{\text {out }}$ of $25 \mu$ s was necessary to account for the reduced rf-set sensor's signal contrast to farther QDs, for which the final visibility is $\sim 64 \%$. However, as shown in Supplementary Note 6, the visibility of the further QDs can be easily enhanced to more than $78 \%$ by simply improving the electronics of the measurement system, for example, with FPGA programming.

To acquire the 2D plots shown in Fig. 3b, e, the typical Ramsey pulse sequence of $\mathrm{I}-\mathrm{W}-\mathrm{O}(\pi / 2)-\mathrm{A}_{\mathrm{ex}}-\mathrm{O}(\pi / 2)-\mathrm{W}-\mathrm{R}$ (Fig. 3b, top 
a
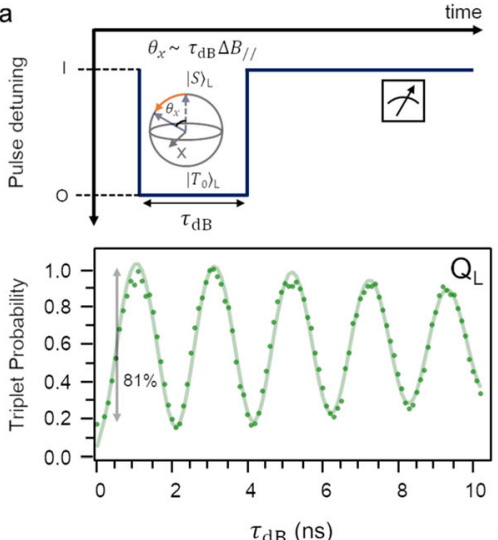

d

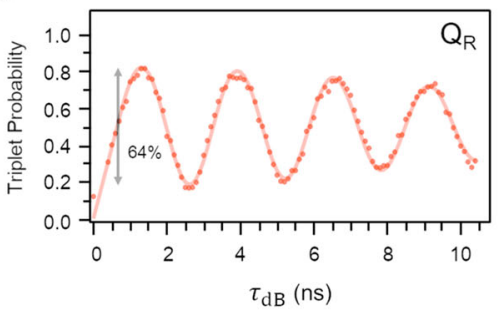

b
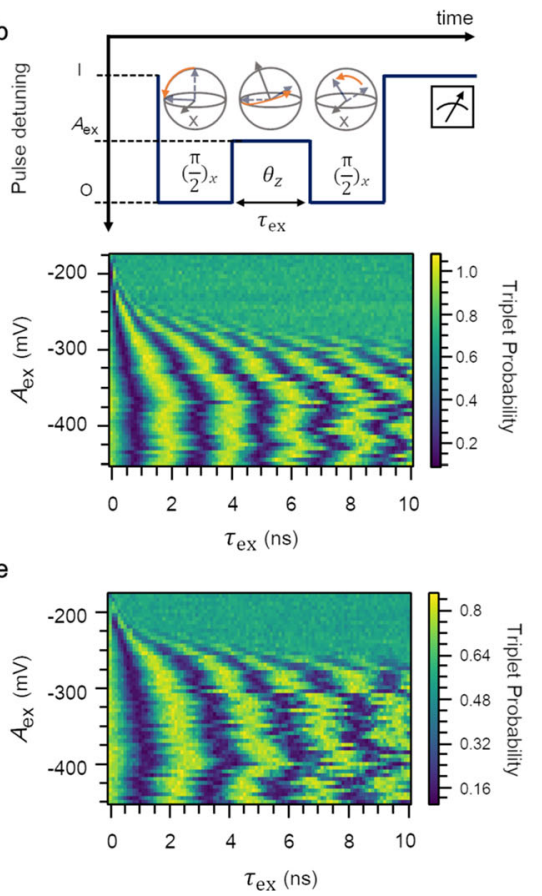
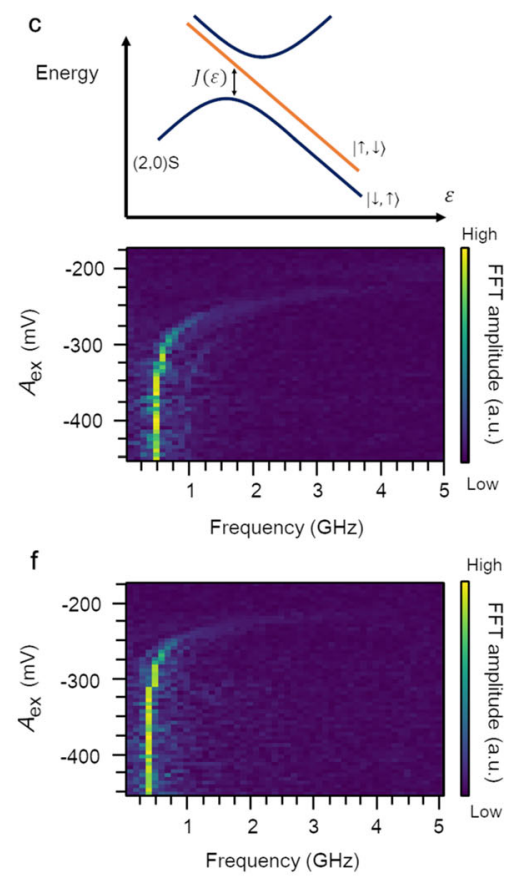

Fig. 3 High-visibility two-axis control of two $\mathbf{S T}_{\mathbf{0}}$ qubits. a, d Coherent $\mathrm{ST}_{0}$ oscillation of $\mathrm{Q}_{\mathrm{L}}\left(\mathrm{Q}_{\mathrm{R}}\right)$ under large $\Delta B / / .81 \%(64 \%)$ quantum oscillation visibility is defined by the initial oscillation amplitude, which is in good agreement with the analytic model with thermal effects and spin relaxation (See Supplementary Note 5). b, e Coherent exchange oscillation and two-axis control of $Q_{L}\left(Q_{R}\right)$ on the Bloch sphere. The top panel of $\mathbf{b}$ shows the Ramsey pulse sequence where the first $\pi / 2$ pulse induces equal superposition of $S$ and $T_{0}$ spin states, and the phase evolution under non-zero $J(\varepsilon)$ is probed by the second $\pi / 2$ pulse. By varying the pulse amplitude $A_{\text {ex }}$ and the evolution time $\tau_{e x}$ at the exchange step, the high-resolution rotation axis evolution and an energy spectrum consistent with the expected functional form of $J(\varepsilon)^{38}$, the schematic of which is shown in the top panel of $\mathbf{c}$, are confirmed by the fast Fourier transform (FFT) plots in $\mathbf{c}, \mathbf{f}$.

panel) was applied, and the detuning amplitude $A_{\text {ex }}$ and evolution time $\tau_{e x}$ at the exchange step were varied. The figures show highvisibility quantum oscillation as well as continuous evolution of rotation axis on the Bloch sphere as $A_{\text {ex }}$ is varied over different regimes, where $T_{2}^{*}$ is limited by the charge noise for $J(\varepsilon)>\Delta B_{\text {// }}$ or by fluctuations in $\Delta B_{/ /}$for $J(\varepsilon) \sim 0$. The fast Fourier transform (FFT) of the exchange oscillations along the exchange detuning axis (Fig. 3c, $\mathrm{f}$ ) confirms the control of the $\mathrm{ST}_{0}$ qubit over the two axes on the Bloch sphere for both $Q_{L}$ and $Q_{R}$, which is consistent with the expected qubit energy splitting (Fig. 3c, top panel). We emphasize that the measurement of two qubits is possible with one accompanied rf-set, which can be useful for the linear extension of the $\mathrm{ST}_{0}$ qubits because the charge sensor does not need to be aligned with the QD array. In this work, we focused on independent two single-qubit gate operation; nevertheless, we expect that long $T_{1}$ at EST readout will allow the sequential measurement of two-qubit states for a given quantum operation, which, in turn, will allow two-qubit correlation measurement, enabling full two-qubit state and process tomography in the future. Characterization of the two-qubit interaction of $\mathrm{ST}_{0}$ qubits in the current quadruple dot array, for example by dipole coupling $^{6,10}$ or exchange interaction ${ }^{36}$, is the subject of current investigations.

\section{DISCUSSION}

High-visibility readout of the $\mathrm{ST}_{0}$ qubit at large $\Delta B_{/ /}$is necessary for high-fidelity $\mathrm{ST}_{0}$ qubit operations ${ }^{6,37}$. We performed highvisibility single-shot readout of two adjacent $\mathrm{ST}_{0}$ qubits at $\Delta B_{\text {// }}$ of $100 \mathrm{mT}\left(\sim 500 \mathrm{MHz} \cdot h \cdot\left(g^{*} \cdot \mu_{B}\right)^{-1}\right)$ by direct EST with one rf-set. No mixing between $T_{0}$ and $(1,1) S$ state was observed at the EST readout point, which would allow sequential readout of multiple arrays of qubits due to the long $T_{1}$. Full one-electron signal difference discriminates the $\mathrm{S}$ and $\mathrm{T}_{0}$ states compared to other readout methods where the dipolar charge difference is measured to readout the $\mathrm{ST}_{0}$ qubit states ${ }^{13,16}$. This feature can be especially useful for scaling up the $\mathrm{ST}_{0}$ qubits for the following reasons: (1) the large signal contrast can result in high visibility and lowmeasurement error, and (2) the sensor does not need to be aligned along the QD array. Especially for GaAs spin qubits, highvisibility $\mathrm{ST}_{0}$ qubit readout allows fast nuclear-spin fluctuation measurements, which will enable accurate feedback/stabilization of the nuclear-spin bath for high-fidelity qubit control ${ }^{2,32,37}$. Furthermore, our method does not require additional metastable states ${ }^{15,17,46}$ or pulsing sequences for high-fidelity measurements at large $\Delta B_{/ /}{ }^{14,16}$, showing that the experimental complexity is greatly reduced. EST readout of $\mathrm{ST}_{0}$ qubits in nuclear-spin-free systems, including $\mathrm{Si}$, may also enhance the measurement fidelity by providing even longer $T_{1}$ for electron spins ${ }^{7,47,48}$. We further expect that the large $\Delta B_{/ /}$based high-fidelity control combined with the high-fidelity readout method will be a powerful tool not only for single-qubit operations but also for exploring the chargenoise insensitive two-qubit operations of the $\mathrm{ST}_{0}$ qubits using extended sweet spot ${ }^{6}$.

Because the highest bandwidth potential of rf-reflectometry cannot be fully exploited with the CDS technique used in this study, we expect that the use of FPGA to detect the peaks from the bare rf-demodulated single-shot traces will enhance the visibility to at least $88 \%(78 \%)$ for $Q_{L}\left(Q_{R}\right)$. The use of FPGA programming will also allow faster nuclear environment Hamiltonian learning ${ }^{37}$, which can be useful in, for example, studying the time-correlation of nuclear-spin bath fluctuations at different QD sites. We have taken the thermal tunneling probabilities into the analysis, and have successfully modeled the coherent $\mathrm{ST}_{0}$ oscillation in our measurement setup, and derived the measurement fidelities. In the future, we plan to improve the performance by adopting an FPGA-based customized measurement, reducing electron temperature, and further optimizing the electronic signal 
path. However, even with the current limitations, the achieved visibility of $81 \%$ for $\mathrm{ST}_{0}$ qubits at large $\Delta B_{/ /}$shows potential to realize high-fidelity quantum measurements in scalable and individually addressable multiple QD arrays in semiconductors.

\section{METHODS}

\section{Device fabrication}

The quadruple QD device was fabricated on a GaAs/AlGaAs heterostructure with a $2 D E G$ formed $73 \mathrm{~nm}$ below the surface. The transport property of the 2DEG shows mobility $\mu=2.6 \times 10^{6} \mathrm{~cm}^{2} \mathrm{~V}^{-1} \mathrm{~s}^{-1}$ with electron density $n=4.6 \times 10^{11} \mathrm{~cm}^{-2}$ and temperature $T=4 \mathrm{~K}$. Mesa was defined by the wet etching technique to eliminate the 2DEG outside the region of interest. Ohmic contact was formed through metal diffusion to connect the 2DEG with the electrode on the surface. The depletion gates were fabricated on the surface using standard e-beam lithography and metal evaporation. The QD array axis was oriented parallel to the [011] crystallographic direction of GaAs. Subsequently, the micromagnet was patterned perpendicular to the QD array using standard e-beam lithography, and a Ni $10 \mathrm{~nm} / \mathrm{Co} 250 \mathrm{~nm} /$ Au $5 \mathrm{~nm}$ was deposited using metal evaporation.

\section{Measurement}

The experiments were performed on a quadruple QD device placed on the $20 \mathrm{mK}$ plate in a commercial dilution refrigerator (Oxford instruments, Triton-500). Rapid voltage pulses generated by Agilent M8195A arbitrary waveform generator (65 GSa/s sampling rate) and stable dc voltages generated by battery-operated voltage sources (Stanford Research Systems SIM928) were applied through bias-tees (picosecond Pulselabs 5546) in the dilution refrigerator before applying the metal gates. An LCresonant tank circuit was attached to one of the ohmic contacts near the rf-set with a resonance frequency of $\sim 110 \mathrm{MHz}$ for homodyne detection. The reflected rf-signal was first amplified at $4 \mathrm{~K}$ with a commercial cryogenic amplifier (Caltech Microwave Research, CITLF2) and then further amplified at room temperature with home-made low-noise amplifiers. Signal demodulation was performed with an ultra-high-frequency lock-in amplifier (Zurich instrument UHFLI), and the demodulated amplitude was processed using a boxcar integrator built in the UHFLI for CDS. The CDS peaks were counted with an external photon counter (Stanford Research, SR400). The pulse parameters could be rapidly swept via a hardware looping technique, which enabled fast acquisition of the $\Delta B_{/ /}$oscillations. In Supplementary Note 8, we show the details of the measurement setup, CDS technique, and signal analysis.

\section{DATA AVAILABILITY}

The data that support the findings of this study are available from the corresponding author upon request.

Received: 3 December 2019; Accepted: 6 July 2020;

Published online: 29 July 2020

\section{REFERENCES}

1. Petta, J. R. et al. Coherent manipulation of coupled electron spins in semiconductor quantum dots. Science 309, 2180-2184 (2005).

2. Foletti, S., Bluhm, H., Mahalu, D., Umansky, V. \& Yacoby, A. Universal quantum control of two-electron spin quantum bits using dynamic nuclear polarization. Nat. Phys. 5, 903-908 (2009).

3. Morello, A. et al. Single-shot readout of an electron spin in silicon. Nature 467, 687-691 (2010).

4. Zajac, D. M. et al. Resonantly driven CNOT gate for electron spins. Science 359, 439-442 (2018).

5. Kim, D. et al. High-fidelity resonant gating of a silicon-based quantum dot hybrid qubit. npj Quantum Inf. 1, 15004 (2015).

6. Nichol, J. M. et al. High-fidelity entangling gate for double-quantum-dot spin qubits. npj Quantum Inf. 3, 3 (2017).

7. Yoneda, J. et al. A quantum-dot spin qubit with coherence limited by charge noise and fidelity higher than 99.9\%. Nat. Nanotechnol. 13, 102 (2018).

8. Veldhorst, M. et al. An addressable quantum dot qubit with fault-tolerant controlfidelity. Nat. Nanotechnol. 9, 981-985 (2014).
9. Kim, D. et al. Quantum control and process tomography of a semiconductor quantum dot hybrid qubit. Nature 511, 70-74 (2014).

10. Shulman, M. D. et al. Demonstration of entanglement of electrostatically coupled singlet-triplet qubits. Science 336, 202-205 (2012).

11. Elzerman, J. M. et al. Single-shot read-out of an individual electron spin in a quantum dot. Nature 430, 431-435 (2004).

12. Meunier, T. et al. High fidelity measurement of singlet-triplet state in a quantum dot. Phys. Status Solidi B 243, 3855-3858 (2006).

13. Barthel, C., Reilly, D. J., Marcus, C. M., Hanson, M. P. \& Gossard, A. C. Rapid singleshot measurement of a singlet-triplet qubit. Phys. Rev. Lett. 103, 160503 (2009).

14. Harvey-Collard, P. et al. High-fidelity single-shot readout for a spin qubit via an enhanced latching mechanism. Phys. Rev. X 8, 021046 (2018).

15. Nakajima, T. et al. Robust single-shot spin measurement with $99.5 \%$ fidelity in a quantum dot array. Phys. Rev. Lett. 119, 017701 (2017).

16. Orona, L. A. et al. Readout of singlet-triplet qubits at large magnetic field gradients. Phys. Rev. B 98, 125404 (2018).

17. Broome, M. A. et al. High-fidelity single-shot singlet-triplet readout of precisionplaced donors in silicon. Phys. Rev. Lett. 119, 046802 (2017).

18. Eng, K. et al. Isotopically enhanced triple-quantum-dot qubit. Sci. Adv. 1, e1500214 (2015).

19. Tracy, L. A. et al. Single shot spin readout using a cryogenic high-electronmobility transistor amplifier at sub-Kelvin temperatures. Appl. Phys. Lett. 108, 063101 (2016).

20. Watson, T. F. et al. A programmable two-qubit quantum processor in silicon. Nature 555, 633-637 (2018).

21. Veldhorst, M. et al. A two-qubit logic gate in silicon. Nature 526, 410-414 (2015).

22. Johnson, A. C., Petta, J. R., Marcus, C. M., Hanson, M. P. \& Gossard, A. C. Singlettriplet spin blockade and charge sensing in a few-electron double quantum dot. Phys. Rev. B 72, 165308 (2005).

23. Johnson, A. C. et al. Triplet-singlet spin relaxation via nuclei in a double quantum dot. Nature 435, 925-928 (2005).

24. Petta, J. R. et al. Pulsed-gate measurements of the singlet-triplet relaxation time in a two-electron double quantum dot. Phys. Rev. B 72, 161301 (2005).

25. Volk, C. et al. Loading a quantum-dot based "Qubyte" register. npj Quantum Inf. 5, 29 (2019).

26. Mills, A. R. et al. Shuttling a single charge across a one-dimensional array of silicon quantum dots. Nat. Commun. 10, 1063 (2019).

27. Martins, F. et al. Negative spin exchange in a multielectron quantum dot. Phys. Rev. Lett. 119, 227701 (2017).

28. Borselli, M. G. et al. Undoped accumulation-mode Si/SiGe quantum dots. Nanotechnology 26, 375202 (2015).

29. Lim, W. H. et al. Electrostatically defined few-electron double quantum dot in silicon. Appl. Phys. Lett. 94, 173502 (2009).

30. Barthel, $C$. et al. Relaxation and readout visibility of a singlet-triplet qubit in an Overhauser field gradient. Phys. Rev. B 85, 035306 (2012).

31. Petta, J. R. et al. Dynamic nuclear polarization with single electron spins. Phys. Rev. Lett. 100, 067601 (2008).

32. Bluhm, H., Foletti, S., Mahalu, D., Umansky, V. \& Yacoby, A. Enhancing the coherence of a spin qubit by operating it as a feedback loop that controls its nuclear spin bath. Phys. Rev. Lett. 105, 216803 (2010).

33. Yoneda, J. et al. Robust micromagnet design for fast electrical manipulations of single spins in quantum dots. Appl. Phys. Express 8, 084401 (2015).

34. Ito, T. et al. Four single-spin Rabi oscillations in a quadruple quantum dot. Appl. Phys. Lett. 113, 093102 (2018).

35. Sigillito, A. J. et al. Site-selective quantum control in an isotopically enriched Si 28 / Si 0.7 Ge 0.3 quadruple quantum dot. Phys. Rev. Appl. 11, 061006 (2019).

36. Noiri, A. et al. A fast quantum interface between different spin qubit encodings. Nat. Commun. 9, 5066 (2018).

37. Shulman, M. D. et al. Suppressing qubit dephasing using real-time Hamiltonian estimation. Nat. Commun. 5, 5156 (2014).

38. Dial, O. E. et al. Charge noise spectroscopy using coherent exchange oscillations in a singlet-triplet qubit. Phys. Rev. Lett. 110, 146804 (2013).

39. Chesi, S. et al. Single-spin manipulation in a double quantum dot in the field of a micromagnet. Phys. Rev. B 90, 235311 (2014).

40. Pioro-Ladrière, $M$. et al. Electrically driven single-electron spin resonance in a slanting Zeeman field. Nat. Phys. 4, 776-779 (2008).

41. Reilly, D. J., Marcus, C. M., Hanson, M. P. \& Gossard, A. C. Fast single-charge sensing with a rf quantum point contact. Appl. Phys. Lett. 91, 162101 (2007).

42. Barthel, $C$. et al. Fast sensing of double-dot charge arrangement and spin state with a radio-frequency sensor quantum dot. Phys. Rev. B 81, 161308 (2010).

43. Hanson, R. et al. Single-shot readout of electron spin states in a quantum dot using spin-dependent tunnel rates. Phys. Rev. Lett. 94, 196802 (2005).

44. Keith, D. et al. Benchmarking high fidelity single-shot readout of semiconductor qubits. N. J. Phys. 21, 063011 (2019). 
45. Kawakami, E. et al. Gate fidelity and coherence of an electron spin in an Si/SiGe quantum dot with micromagnet. Proc. Natl Acad. Sci. USA 113, 11738-11743 (2016).

46. Studenikin, S. A. et al. Enhanced charge detection of spin qubit readout via an intermediate state. Appl. Phys. Lett. 101, 233101 (2012).

47. Huang, W. et al. Fidelity benchmarks for two-qubit gates in silicon. Nature $\mathbf{5 6 9}$ 532-536 (2019).

48. Kawakami, E. et al. Electrical control of a long-lived spin qubit in a Si/SiGe quantum dot. Nat. Nanotechnol. 9, 666-670 (2014).

\section{ACKNOWLEDGEMENTS}

This work was supported by Samsung Science and Technology Foundation under Project Number SSTF-BA1502-03. We thank the Mark Eriksson group for their technical support on the circuit board used in the experiment.

\section{AUTHOR CONTRIBUTIONS}

D.K. and W.J. conceived the project, performed the measurements, and analyzed the data with the help of S.P. J.K. fabricated the device with the help of H.C., J.E., and Y.C. M.C. and W.J. built the experimental setup and configured the measurement software. V.U. synthesized and provided the GaAs heterostructure. All authors contributed to the preparation of the manuscript.

\section{COMPETING INTERESTS}

The authors declare no competing interests.

\section{ADDITIONAL INFORMATION}

Supplementary information is available for this paper at https://doi.org/10.1038/ s41534-020-00295-w.

Correspondence and requests for materials should be addressed to D.K.

Reprints and permission information is available at http://www.nature.com/ reprints

Publisher's note Springer Nature remains neutral with regard to jurisdictional claims in published maps and institutional affiliations.

Open Access This article is licensed under a Creative Commons Attribution 4.0 International License, which permits use, sharing, adaptation, distribution and reproduction in any medium or format, as long as you give appropriate credit to the original author(s) and the source, provide a link to the Creative Commons license, and indicate if changes were made. The images or other third party material in this article are included in the article's Creative Commons license, unless indicated otherwise in a credit line to the material. If material is not included in the article's Creative Commons license and your intended use is not permitted by statutory regulation or exceeds the permitted use, you will need to obtain permission directly from the copyright holder. To view a copy of this license, visit http://creativecommons. org/licenses/by/4.0/.

(c) The Author(s) 2020 